\title{
Haemopneumothorax and Mediastinal Emphysema A Dramatic Rescue in the South Atlantic
}

\author{
Major J B L Kabuubi \\ MB, BS, Acad Dip Biochem, MRCS, MRCP, RAMC \\ Consultant Physician \\ The Princess Mary's Royal Air Force Hospital, Akrotiri, BFPO 57
}

SUMMARY: Spontaneous haemopneumothorax has a high mortality and morbidity which increase if the treatment is delayed. A patient with tension haemopneumothorax and mediastinal emphysema, rescued from a fishing boat in the middle of the South Atlantic, responded well to conservative treatment, despite delay in initiating treatment. The clinical presentation and management are discussed.

\section{Introduction}

The aeromedical rescue of a Russian engineer from a fishing boat off the Falkland Islands at the end of 1986 aroused much international media interest on New Year's Day 1987. The patient arrived at the British Military Hospital Falkland Islands (BMHFI) by a Sea King helicopter over 18 hours after the onset of symptoms. When the distress call was received the exact location of the boat was not known but it was outside the Fishing Exclusion Zone, a distance of about 200 miles from the Falkland Islands. Because of this uncertainty a Royal Fleet Auxiliary (RFA) ship and a Hercules air tanker were used for possible refuelling of the Sea King search and rescue helicopter. The aeromedical rescue team had a working diagnosis of perforated duodenal ulcer, made by the resident Russian doctor. It was observed in transit that the patient was very breathless on lying flat, relieved by sitting up. He was given oxygen throughout the journey. The diagnosis of tension haemopneumothorax with pneumomediastinum was made on arrival at BMHFI.

\section{History}

The history was not immediately available on arrival at BMHFI. Two days after admission the patient was able to give a definitive history through an interpreter: aged 28 , he worked as an engineer, i.e. an officer, on a Soviet fishing ship. He had never been ill before sailing to the South Atlantic. Although he smoked 30 cigarettes a day he had no cough. He gave no relevant past medical history. Prior to the present illness he did not participate in any contact sport or diving and gave no recent history of direct or indirect body trauma. He drank alcohol only moderately. While doing a non-physical job on the ship, without straining, he developed sudden right hypochondrial pain radiating to the right shoulder and became very breathless at rest. From that time he could not remember anything until 48 hours after admission to BMHFI. A resident Russian doctor saw him and made a clinical diagnosis of perforated duodenal ulcer. She put him to bed until the arrival of the aeromedical rescue from the Falkland Islands.

\section{Condition on Admission}

On examination on arrival at BMHFI, he was seme conscious, cold, clammy, orthopnoeic, tachypnoeiz centrally and peripherally cyanosed, and in agony. The pulse was $120 / \mathrm{min}$ regular, BP $110 / 50$. There was \& mediastinal shift to the left (apex beat and trache displaced to the left). He was clinically anaemic and th $\vec{f}$ haemoglobin on admission was $8.9 \mathrm{gram} / \mathrm{d} 1$. There wa resonance on percussion, absent breath sounds over the whole right lung field and subcutaneous emphysematort the right, the left side of the neck and on the left up if chest. Hamman's sign (cardiac crunch) was negator There was no tenderness in the abdomen and $\$$ no organomegally. There was no evidence of recent bơdy trauma. A supine portable chest X-ray showed a righe pneumothorax with complete collapse of the right and shift of the mediastinum to the left, with air alsng the fascial planes from both sides of the mediastinurit left and right side of neck. There was a diffuse opacity the left upper/middle zone, partly due to engorgemeng of pulmonary vessels and subcutaneous emphysema oo the left upper chest. (X-rays taken immediately after insertion of the chest drain showed clearing of left uppeg middle zone). There was no visible fluid level. A chest drain with an underwater seal was inserted in the rigf fifth intercostal space in the mid axillary line and immediately $1840 \mathrm{ml}$ of frank blood drained off. The was immediate improvement of symptoms and signe breathlessness, pain, cyanosis, and tachycardia. The clotting screen was normal. He was transfused four unitos of fresh blood. Twelve hours after admission $2516 \mathrm{ml}$ 新 blood had drained from the right haemothorax. By the no more blood was draining and the lung had started te. expand. Seventy $\mathrm{ml}$ of frank blood was aspirated from the left side. Forty-eight hours after admission he was fully conscious and felt subjectively well. Culture of the blood from the chest was sterile. The right lung was ver slow to expand so the drain was not removed until th sixth day and by then the lung had expanded up to the clavicle. He developed low grade fever and right bas atelectasis and was given Amoxil and physiotherapy. Ho was mobilised and on the sixteenth day he was able to 
leave the Falkland Islands on the aeromedical flight via Brize Norton to the Soviet Union. The lung had clinically nearly fully expanded but radiologically there was some fluid in the right fissure with loss of volume on the right lower lobe. He was still taking Amoxil. The final outcome of his progress in the Soviet Union is not known. The final diagnosis was spontaneous tension haemopneumothorax, with subcutaneous emphysema secondary to mediastinal emphysema.

\section{Discussion}

The delay of more than 18 hours between receiving the distress call and bringing in the casualty was due to the logistics. Permission had to be given by the Headquarters British Falkland Islands to allow the operation to begin. The RFA ship had to sail from the Falkland Islands, to be in position for possible refuelling. Co-ordination had to be made with the air tanker. The operation worked well without a hitch.

The clinical diagnosis of tension pneumothorax with mediastinal emphysema was not in doubt but the haemothorax was rather unexpected. No fluid was detected clinically. He was too ill to sit up so he was examined lying down on a stretcher, rolling to one side then to the other. Only supine portable chest $\mathrm{x}$-rays were available therefore no fluid level was seen. There was no clinical or radiological evidence of trauma by a sharp or blunt instrument and no evidence of trauma during winching (winching and car seat injuries can rarely cause trauma to the great vessels). Patients with air flow obstruction such as asthma, bronchiolitis or whooping cough may develop spontaneous pneumothorax after severe straining, in labour or during severe coughing ${ }^{1}$. Sarcoid interstitial lung disease with non-caseating granuloma is a rare cause of pneumothorax. Gomm ${ }^{2}$ described a case of spontaneous haemopneumothorax in early sarcoidosis without radiological evidence of lung infiltration. Tuberculosis and malignant disease should be ruled out. In the patient presented there was no history or clinical evidence of underlying lung disease and although a heavy smoker he had no cough. Mediastinal emphysema may accompany spontaneous pneumothorax in presence of tension pneumothorax ${ }^{1}$. To get both haemopneumothorax and mediastinal emphysema one can postulate that the patient had a spontaneous rupture of a mediastinal rather than an apical bullous lesion but the two lesions could not be ruled out. The subpleural air from the ruptured apical bulla or bleb could have "dissected" towards the hilum ${ }^{3}$ but the severity of the subcutaneous emphysema implies sudden pressure in the mediastinum. Hamman's sign (clicking, crunching or grating sounds synchronous with heart beat) used to be regarded as diagnostic of mediastinal emphysema; it is said to occur in $50 \%$ of cases but also in left pneumothorax without mediastinal emphysema, bullous emphysema of the lingula, dilated lower oesophagus, pneumoperitoneum with high diaphragm and gastric dilatation. It is best heard in expiration leaning to the left, but may be difficult to hear ${ }^{1}$. Usually in mediastinal emphysema air is reabsorbed spontaneously within a week, but an underwater drainage may be inserted if necessary. In a large series of 72 patients with spontaneouso pneumothorax Lindskog described 2 patients who had massive bleeding of $6300 \mathrm{ml}$ and $4700 \mathrm{ml}$ respectively; both responded to conservative treatment (see below) and 5 patients, i.e. $7 \%$, had pneumomediastinum ando pneumothorax ${ }^{3}$. Spontaneous pneumothorax is a을 disease of young men between the ages of 15-45 years $\frac{\overline{\bar{S}}}{\overline{5}}$ with a male to female ratio of $15: 1$ and an incidence of $2-5 \%$ in patients with spontaneous pneumothorax ${ }^{4}$. As? the lung collapses there is a tear in the arterioles in the adhesions between the parietal and visceral pleura, $\overrightarrow{0}$ followed by small subpleural bronchial vessels in the $\overrightarrow{\vec{\omega}}$ ruptured bullae 5 .

Because the arterioles are supplied at systemic pressure, the bleeding can be severe. These arterioles do not have any muscular component, therefore there is? no vasoconstriction to stop the bleeding ${ }^{6}$. The negative intrathoracic pressure, together with the above factors, $\dot{ }$ explains why the bleeding may be massive. The symptoms of hypovolemia and respiratory embarrassment in haemopneumothorax might mimic오 either myocardial infarct, pulmonary embolism, rupture of major chest organs (heart, great vessels, oesophages ${ }_{\mathbb{\Phi}}^{7}$ or trachea) or intra-abdominal emergencies such @s perforated intestinal viscus or acute pancreatitis ${ }^{7}$. Bn traumatic haemophneumothorax, injury to abdomingr viscera should be looked for, especially in the presen $6 \overrightarrow{0}$ of a diaphragmatic tear. Thoracoscopy and peritoneog lavage should be done in traumatic haemopneumethorax due to penetrating chest wounds below the four and fifth intercostal space, to look for the presence or absence of diaphragmatic tear, therefore avoiding unnecessary laparotomy. Patients with tension $\mathbb{\perp}$ pneumothorax usually do not develop pneumoperito- $\stackrel{\varrho}{\Rightarrow}$ neum $^{8}$. It tends to occur in patients with multiple injuries $\overrightarrow{\overrightarrow{0}}$ and pneumothorax treated with mechanical ventilation using positive end-expiratory pressure. In our patient: there was no evidence of intestinal pneumatosis. The initial acute severe right hypochondrial pain radiating to 3 . the right shoulder was probably due to sudden increased tension in the chest pushing the liver downwards.

Axillary intercostal drainage caused a dramatic improvement of symptoms and signs in our patient. $2516 \frac{\widetilde{J}}{\mathrm{~F}}$ $\mathrm{ml}$ of blood draining out of the right pleural space was a: considerable haemorrhage but, reviewing the literature, 3 . $8000 \mathrm{ml}$ has been aspirated from one hemithorax in spontaneous haemopneumothorax in 18 hours ${ }^{9}$. There was unease among the bedside assistants because of the $\mathrm{O}$ quantity of blood draining out of the chest, but the patient improved as more blood drained out. The chesto drain was inserted in the fifth intercostal space in midaxillary line, so that chances of puncturing the liver were $\mathscr{\sigma}$ remote. Bleeding from an anomalous intercostopul- $N$ monary artery may occur during the introduction of $\mathrm{w}_{\mathrm{W}}$ 
chest drain, but never stops spontaneously and a thoracotomy is done as an urgent measure to stop the bleeding ${ }^{10}$. It can be excluded by angiography. This was ruled out in the patient presented because he improved dramatically on draining the chest. Seventy $\mathrm{ml}$ of blood aspirated from the left pleural space was due to blood tracking from right to the left because the left hemithorax remained normal throughout the time of observation. The aim of the clinical management of a patient with haemopneumothorax is a rapid adequate chest decompression and blood transfusion. Delay in draining a haemothorax can cause organisation of the blood clot into a mass in the pleural cavity. Willson ${ }^{11}$ described a case of delayed spontaneous haemopneumothorax with a radiological apical mass in the thoracic cavity confirmed by ultrasound. A thoracotomy had to be done to exclude a sinister pathology. Because of defibrinating action of cardiorespiratory movements depositing fibrin on the pleural surface, the blood usually remains in a fluid state. The fluid or blood may become organised into a fibrin body or "pleural mouse" which appears as an oval opacity lying free in the pleural cavity simulating an intrapleural mass on a PA and lateral chest radiograph ${ }^{11}$. In the past two drains have been advocated: one anteriorly in the second intercostal space in the mid clavicular line to drain air and the second one at the base posteriorly to drain fluid. The first can cause damage to the pectoralis major in men, the breast in women, as well as damage to nerve plexus and subclavian vessels. The basal site may cause perforation of the diaphragm and damage to the spleen, liver, stomach and other intra-abdominal pathology ${ }^{12}$. Three chest drains have also been used by others. The general concensus today is that a single wide-bore mid-axillary drain at the fourth or fifth intercostal space is adequate in treating both traumatic and spontaneous haemopneumothorax ${ }^{12}$. Repeated chest aspiration (thoracocentesis) through the base is indicated if the haemothorax is small or as an adjunct to axillary drainage. A suction pump with a negative pressure of $20 \mathrm{~cm}$ of water is advocated by some ${ }^{7}$ but it is not always necessary. Thoracotomy and a probable decortication is indicated in the following circumstances:

1. When the diagnosis is delayed; blood clots form. This can cause lung fibrosis and constriction ${ }^{4}$. Decortication is mandatory in presence of scar tissue on the pleural membranes.

2. In presence of persistent bleeding. The bleeding sites must be identified and secured and blood clots evacuated from the pleural cavity.

3 . In the presence of bronchopulmonary fistula. To seal air leakage from lung surface by resection of areas of emphysematous bullae .

4. In presence of a mass in pleural cavity: to exclude pleural malignancy, sarcoidosis and tuberculosis ${ }^{2}$.

5. Failure to respond to conservative management.

Whether decortication lobectomy, or wedge resection is done depends on the thoracotomy findings. The presence of subcutaneous emphysema may have significance to the outcome of the management spontaneous haemopneumothorax. Its presence mas however, indicate that the pneumothorax has decon pressed externally ${ }^{13}$.

The final outcome of the progress of our patient is not known. On leaving the Falkland Islands he was well, buㅐㅐ the chest X-rays showed some atelectasis and fluid in the transverse fissure although the lung had near completely expanded. A long follow up for at least year is required to exclude underlying lung disease andब recurrence of symptoms which can occur weeks or eved months after the initial presentation. The mortality of haemopneumothorax varies between $27 \%$ to $15 \%$ b $\vec{\Phi}$

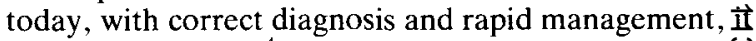
is in single figures ${ }^{4}$. The aeromedical rescue team equipped to initiate treatment in such an emergenc. The doctor remained airborne during the rescue. He could not examine the patient properly on board because of the noise inside the helicopter which cay easily exceed $100 \mathrm{db}^{14}$. As the patient was stable he exercised caution, and his judgement not to intervent: while in flight was correct. To intervene without back-upi facilities could spell disaster. The effect of altitude of tension pneumothorax is that the pressure remains the same in the affected pleural cavity, but the redused ambient pressure causes a reduction in the pressurs the left hemithorax compared with ground/sea lese causing an increase in the shift to the left. The effect 8 f helicopter flying at a maximum altitude of $1,000 \mathrm{ft}$. $\overrightarrow{\mathrm{as}}$ minimal. This is the probable time sequence of 918 events in our patient: he developed spontaneous ruptive of a mediastinal or apical lung bulla, causing tengion pneumothorax and mediastinal emphysema, followeel by intrapleural bleeding and subcutaneous emphysemo The increased intrathoracic pressure due to tensio pneumothorax may have exerted direct pressure to the्टि bleeding vessels to check the bleeding, and the release of air from the mediastinum through the chest wall and neck (subcutaneous emphysema) may have modifie both the pneumothorax and pneumomediastinum. A the above forces balanced out, enabling the patient survive, despite the delay in initiating treatment and the long evacuation chain. If the patient had not had subcutaneous emphysema the prognosis might hake been different. Without the participation of man individuals on land, air and sea, this rescue could nef have been possible.

I am grateful to the RAM College Librarians for the literature search and to Mrs Richards for typing the manuscript.

\section{REFERENCES}

1. Crofton $J$ and Douglas A. Respiratory Diseases, $2 \overline{\overline{\mathrm{m}}}$ Edition, 1975, 478-486.

2. Gомm S A. An unusual presentation of Sarcoidosisn Spontaneous Haemopneumothorax. Postgrad Med nิ. 1984; 60:621-623. 
3. Lindskog $G$ E and Halasz $N$ A. Spontaneous Pneumothorax: A consideration of the pathogenesis and management with a review of 72 hospitalised cases. $A M A$ Archives of Surgery. 1957; 75: 693-698.

4. WhITFORD P and Russel C F J. Spontaneous Haemopneumothorax. Ulster Med J. 1985; 54(1): 72-74.

5. CALVERT R J and SMITH E. An analytic review of spontaneous haemopneumothorax. Thorax. 1955; 10: 64 72 .

6. DEATON W R and JOHnston F R. Spontaneous Haemopneumothorax. I Thorac Cardiovasc Surg. 1962; 43: 413-415.

7. ABYHOLM F E and STOREN G. Spontaneous Haemopneumothorax. Thorax. 1973; 28: 376-378.

8. SCHEIN M and Schwarz H A. Delayed pneumoperitoneum following traumatic haemopneumothorax - A case report. S Afr Med J. 1984; 65(2) 819-820.
9. Mills M and BaisCH B F. Spontancous pneumothorax. Ann Thorac Surg. 1965; 1: 286.

10. NELSON R J. Post operative haemopneumothorax arising from an anomalous intercosto-pulmonary artery. Thorax, 1983: 38: 863-864.

11. Willson S A, Sawicka E $\mathrm{H}$ and Mrtchell C, Spontaneous pneumothorax: An unusual radiological appearance. Brit J Radiol. 1985; 58: 173-175.

12. Melissas J, Diamantis I and Mannell A. Axillary intercostal drain in traumatic haemothorax. $S$ Afr Med $J$. 1986; 70: 588-589.

13. MuCKART D J J. Delayed pneumothorax and haemothorax following observation for stab wounds of the chest. Injury. $1985 ; 16(4): 247-248$

14. Cottrell J J and Garrad C. Emergency Transport by Aeromedical blimp. Br Med J. 1989; 298: 869-870

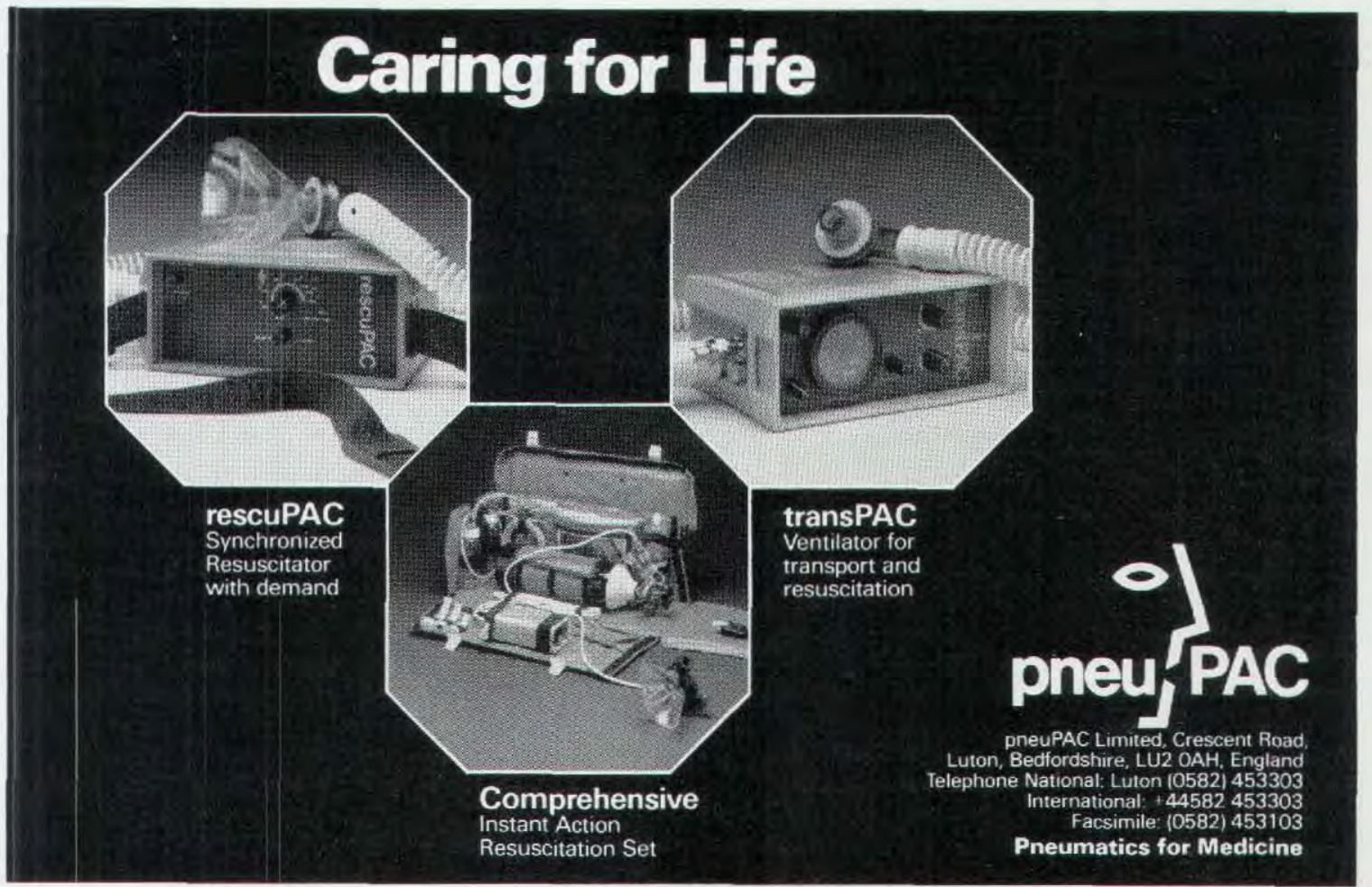

